03

\title{
Теневой фоновый метод - оптический метод исследования ударных волн
}

\author{
(C) С.И. Герасимов, ${ }^{1,2,3,4}$ Н.А. Трепалов ${ }^{1}$ \\ ${ }^{1}$ Российский федеральный ядерный центр - ВНИИ экспериментальной фризики, \\ 607190 Саров, Нижегородская обл., Россия \\ 2 Саровский фризико-технический институт Национального исследовательского ядерного университета „МИФИ“, \\ 607190 Саров, Нижегородская обл., Россия \\ ${ }^{3}$ Национальный исследовательский ядерный университет „МИФИ“, \\ 115409 Москва, Россия \\ ${ }^{4}$ Нижегородский государственный технический университет им. Р.Е. Алексеева, \\ 603950 Нижний Новгород, Россия \\ e-mail: s.i.gerasimov@mail.ru
}

(Поступило в Редакцию 4 апреля 2017 г.)

Приведены результаты применения теневого фонового метода в области диагностики воздушных ударных волн. Продемонстрирована возможность применения теневого фонового метода для визуализации ударных волн, возникающих как при взрыве, так и при движении тел со сверхзвуковыми скоростями. Получаемые данные регистрации позволяют проследить за распространением ударной волны в пространстве, а также в случае взрыва оценить степень асимметрии взрывного энерговыделения и определить параметры ударной волны.

DOI: 10.21883/JTF.2017.12.45201.2279

\section{Введение}

Одной из задач оптической регистрации быстропротекающих процессов, является регистрация ударных волн (УВ), возникающих как при взрыве, так и при движении тел со сверхзвуковыми скоростями. Экспериментальные исследования быстропротекающих процессов требуют организации измерений с высоким временным разрешением. Оптические методы измерений и исследований, имея крайне малый теоретический предел инерционности, представляют собой подходящие инструменты для применения в физике импульсных, быстропротекающих и высокоэнергетических процессов. Важным достоинством является также то, что оптические методы, как правило, являются панорамными методами, собирающими данные одновременно с целой области течения вместо одной точки.

Развитие цифровых методов хранения и обработки данных, а позднее - цифровой фото- видеорегистрации существенно расширило возможности оптических методов, сделало их более удобными для экспериментального применения. Применение цифровой обработки к полученным результатам в настоящее время позволяет существенно увеличить качество и количество получаемых данных об изучаемых физических явлениях. Одним из таких примеров является теневой фоновый метод (ТФМ), в англоязычной литературе он известен как Background Oriented Schlieren (BOS) [1]. ТФМ нашел широкое применение в различных областях научных исследований [2], поскольку позволяет получать качественные и в некоторых случаях количественные поля термодинамических параметров при значительно упрощенной по сравнению с теневыми методами $[3,4]$ аппаратной части. Метод не требует использования оптических элементов, сравнимых по размерам с исследуемым течением. Это свойство делает его удобным для различных полевых исследований и экспериментов и других случаев, когда требуется визуализация течений большого масштаба. В работе продемонстрирована данная особенность ТФМ на примерах визуализации УВ, образованной при движении тел со сверхзвуковой скоростью, и УВ от взрыва. В случае распространения сферической УВ от взрыва продемонстрирована возможность определения ее параметров по результатам ТФМ регистрации.

\section{Теневой фоновый метод}

Возможность применения ТФМ для исследования УВ обусловлена зависимостью коэффициента преломления газов от их плотности и, как следствие, от давления на фронте УВ. Для неионизированных газов с большой точностью выполняется соотношение Гладстона-Дейла

$$
n=1+G \rho,
$$

где $n$ - показатель преломления, $G$ - постоянный для данного газа и данной длины волны коэффициент (постоянная Гладсона-Дейла), $\rho$ - плотность.

Схема регистрации с помощью ТФМ приведена на рис. 1 [5]. В плоскости $\xi \eta$ находится плоская случайная картина, играющая роль фонового экрана. Плоскость $x y$ - плоскость регистрации, в ней расположена приемная матрица видеокамеры. $O S$ - оптическая система с фокусным расстоянием $f$, формирующая 


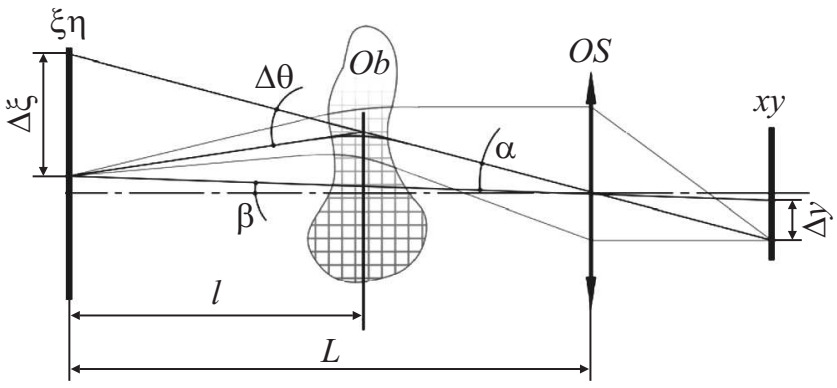

Рис. 1. Траектория луча, проходящего через оптическую неоднородность.

изображение, $O b-$ плоскость, в окрестности которой расположен исследуемый объект. Метод чувствителен к компонентам градиента показателя преломления объекта, перпендикулярным лучу зрения. Отклонение луча $\Delta \theta$, вызванное прохождением его через среду, содержащую оптическую неоднородность, приводит к смещению в плоскости приемной матрицы изображения соответствующего участка случайной картины на $\Delta x$ и $\Delta y$. На регистрации $\Delta x$ и $\Delta y$ основан ТФМ. Распространение луча через неоднородность в плоскости $Z 0 Y$ описывается соотношениями (2), (3)

$$
\begin{gathered}
\Delta \theta=\int_{0}^{S} \frac{1}{n} \frac{\partial n}{\partial y} d z, \\
\Delta y^{\prime}=\int_{0}^{S}\left(\int_{0}^{S} \frac{1}{n} \frac{\partial n}{\partial y} d z\right) d S,
\end{gathered}
$$

где $\Delta y^{\prime}-$ смещение и $\Delta \theta-$ отклонение луча на выходе из неоднородности. Интегрирование производится по пути луча внутри неоднородности $(S)$.

В предположении, что угол $\alpha$ мал, а объект можно считать локализованным в плоскости $O b$, по смещению $\Delta y$ можно из геометрии схемы рассчитать угол $\Delta \theta$ :

$$
\Delta \theta \approx \Delta y \frac{L-f}{l f} \cos ^{2} \beta .
$$

Угол $\beta$ обычно мал, и в большинстве случаев множитель $\cos ^{2} \beta$ стремится к единице и может быть опущен.

Приведенная схема регистрации и соотношения (1)-(4) показывают, что чувствительность схемы ТФМ при фиксированных значениях регистрируемой оптической неоднородности (УВ с определенным значением $\Delta P)$ зависит от параметров схемы регистрации $(L$, $l, f)$. Минимальное регистрируемое значение $\Delta y$ в случае кросс-корреляционной обработки определяется параметрами экрана и физическим размером пикселя.

Визуализация УВ с помощью ТФМ осуществляется на основе регистрации двух и более снимков фонового экрана, с высоким оптическим контрастом и структурой. Одно изображение фонового экрана регистрируется при отсутствии УВ в кадре, другие - при наличии УВ. Последующий программный анализ полученных изображений позволяет визуализировать УВ. В качестве фонового экрана могут использоваться как специально сгенерированные, так и естественные природные фоны [6].

\section{Визуализация ударных волн с помощью теневого фонового метода}

Направление области применения ТФМ в качестве инструмента визуализации оптических неоднородностей (УВ, в частности) условно можно разбить на два направления. Первое направление объединяет в себе схемы оптической регистрации с применением специально созданных фоновых экранов с заранее подобранными оптимальными параметрами (размер и величина разброса зерна) для достижения положительного результата визуализации интересующей оптической неоднородности. На рис. 2 приведен один из таких экранов. Во втором направлении в качестве фоновых экранов используются естественные фоны (лес, облака, трава, песок и т.д.). Ниже представлены результаты визуализации УВ на фоне специальных экранов (рис. 3-6) и на фоне естественных фонов (рис. 7-8).

На рис. 3 представлен фрагмент высокоскоростной видеорегистрации движения пули патрона $7.62 \times 54 R$ ПС, выпущенной из снайперской винтовки Драгунова (СВД), и результат визуализации УВ, вызванной движением пули со сверхзвуковой скоростью. Видеорегистрация осуществлялась на расстоянии порядка $15 \mathrm{~m}$ от СВД. Скорость движения пули в зоне видеорегистрации составила $775 \mathrm{~m} / \mathrm{s}$.

На рис. 4,5 представлены фрагменты визуализации картины процесса выстрела из крупнокалиберного пулемета Владимирова танкового (КПВТ) и пороховой баллистической установки калибра 14.5 (ПБУ-14.5). На приведенных рисунках видны фронт дульной УВ и фронт головного скачка уплотнения. Скорость метаемого объекта $(\mathrm{MO})$ в зоне регистрации составила $998 \mathrm{~m} / \mathrm{s}$ (КПВТ) и $1695 \mathrm{~m} / \mathrm{s}$ (ПБУ-14.5).
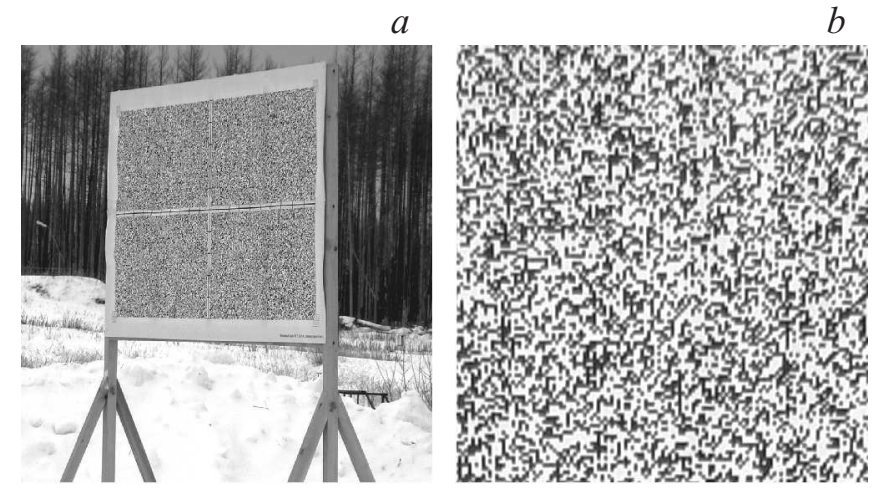

Рис. 2. Внешний вид фонового экрана $(a)$ и увеличенный фрагмент $(b)$. Размер рабочей зоны экрана $2 \times 1 \mathrm{~m}$. 

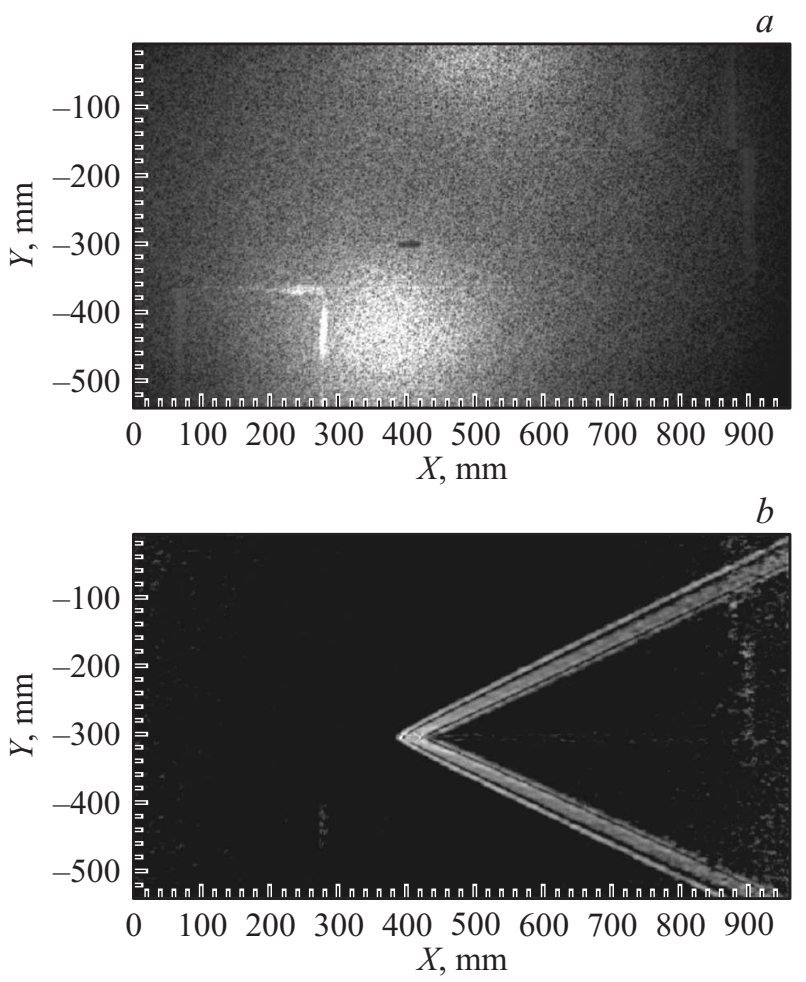

Рис. 3. Исходное изображение $(a)$ и результат визуализации УВ $(b)$.
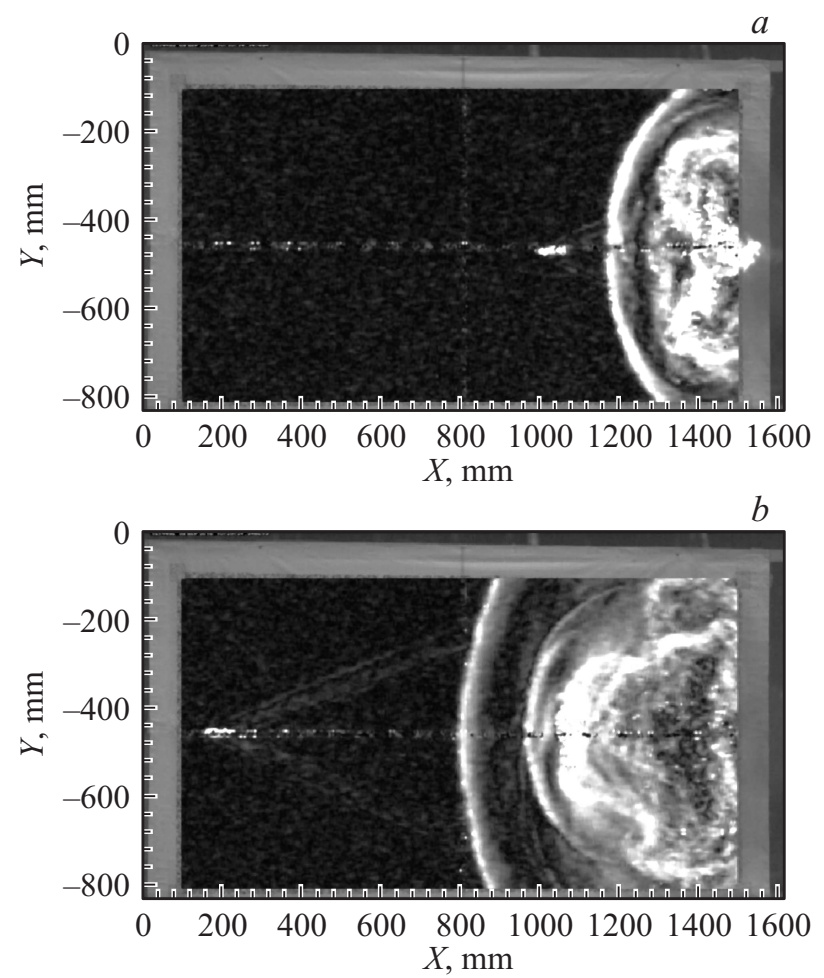

Рис. 4. Визуализация УВ при выстреле из КПВТ.

Форма зарядов взрывчатого вещества (ВВ), соответствующая сфере или полусфере, встречается редко, за исключением специальных постановочных опытов.
Чаще всего форма зарядов представляет собой цилиндр длиной $L$ и диаметром $D$, т.е. цилиндр с удлинением $L / D>1$. В общем случае форма ударной волны (УВ) при взрыве цилиндрического заряда $\mathrm{BB}$ не сферична на достаточно большом удалении от источника взрыва. На рис. 6,7 приведены фрагменты видеорегистрации распространения УВ от зарядов ВВ цилиндрической формы. На рис. 6 представлена визуализация УВ от взрыва заряда ВВ, находящегося в тонкостенной пластиковой цилиндрической оболочке с удлинением заряда 1.8. Заряд представлял собой сборку из ВС-2 [7] и ТЭН $(5, \mathrm{~g})$. На рис. 7 представлена визуализация УВ от взрыва заряда ВВ (ПВВ-7 массой 200 g.) цилиндрической формы с удлинением $L / D=4.3$. Заряд располагался в центре зоны видеорегистрации и устанавливался цилиндрической поверхностью на деревянной подставке, ось заряда перпендикулярна оси видеорегистрации. Результаты визуализации показывают неравномерное распространение УВ на начальном этапе движения и при дальнейшем распространении ее форма постепенно приближается к сферической.

Постановка опытов на ракетно-катапультирующей установке, чаще именуемой ракетным треком, включает разгон объектов испытания (ОИ) по рельсовым направляющим с помощью так называемых ракетных поездов. Разгон ОИ по рельсовым направляющим ракетного трека сопровождается рядом эффектов, требующих качественной визуализации для последующего учета при анализе результатов каждого такого испытания. На
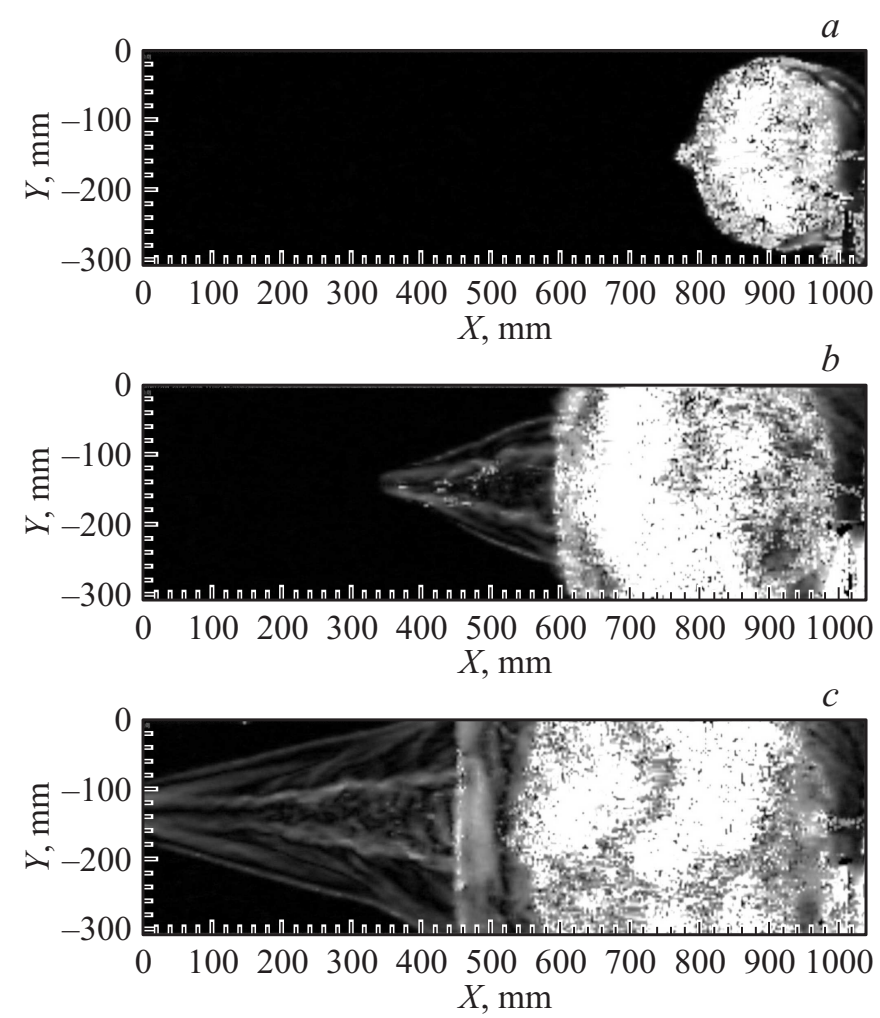

Рис. 5. Визуализация УВ при выстреле из ПБУ-14.5 через $0.25,0.5$ и $0.75 \mathrm{~ms}$ после инициирования заряда. 

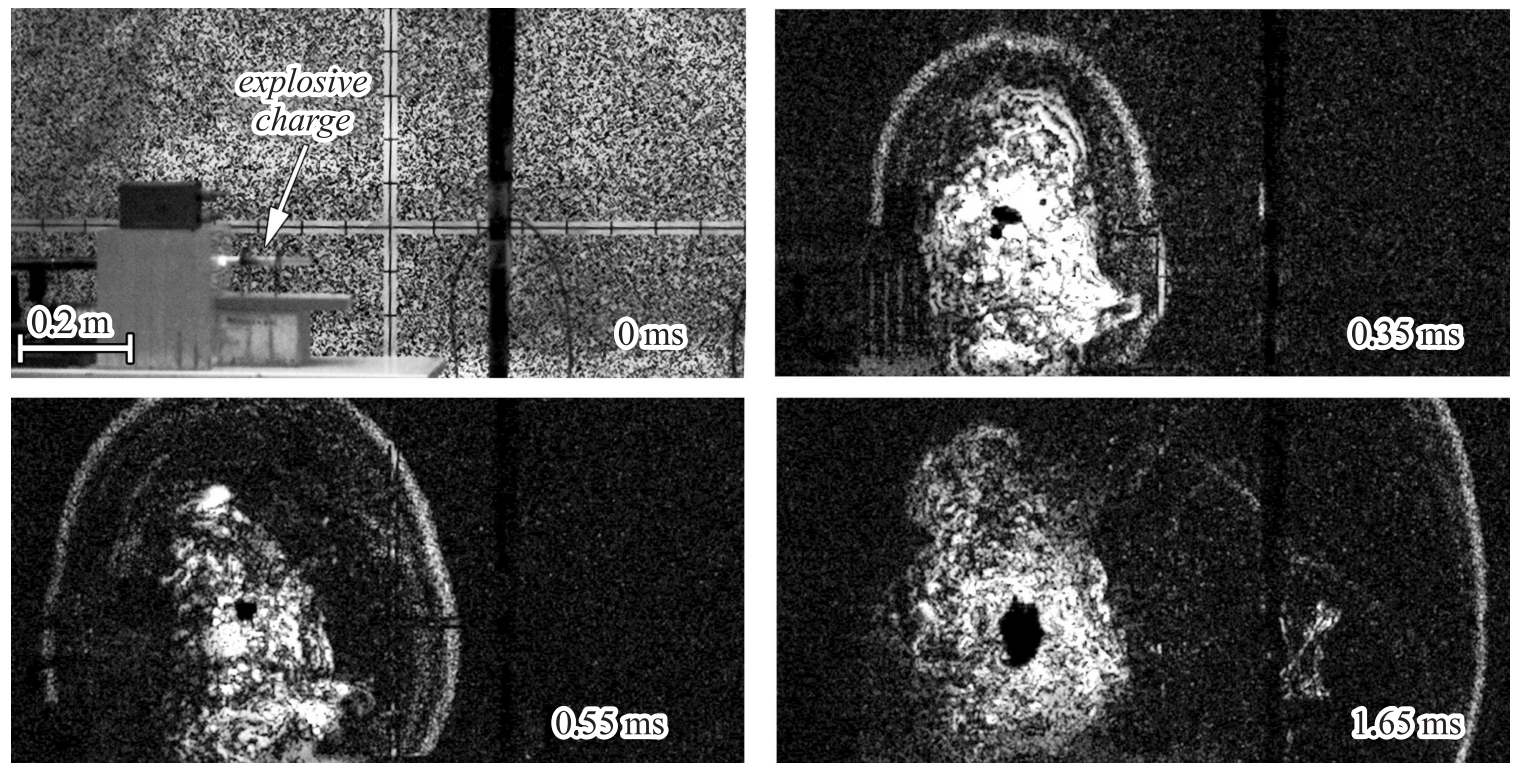

Рис. 6. Регистрация взрыва заряда ВВ цилиндрической формы с удлинением 1.8 через 0, 0.35, 0.55, 1.65 ms после инициирования.
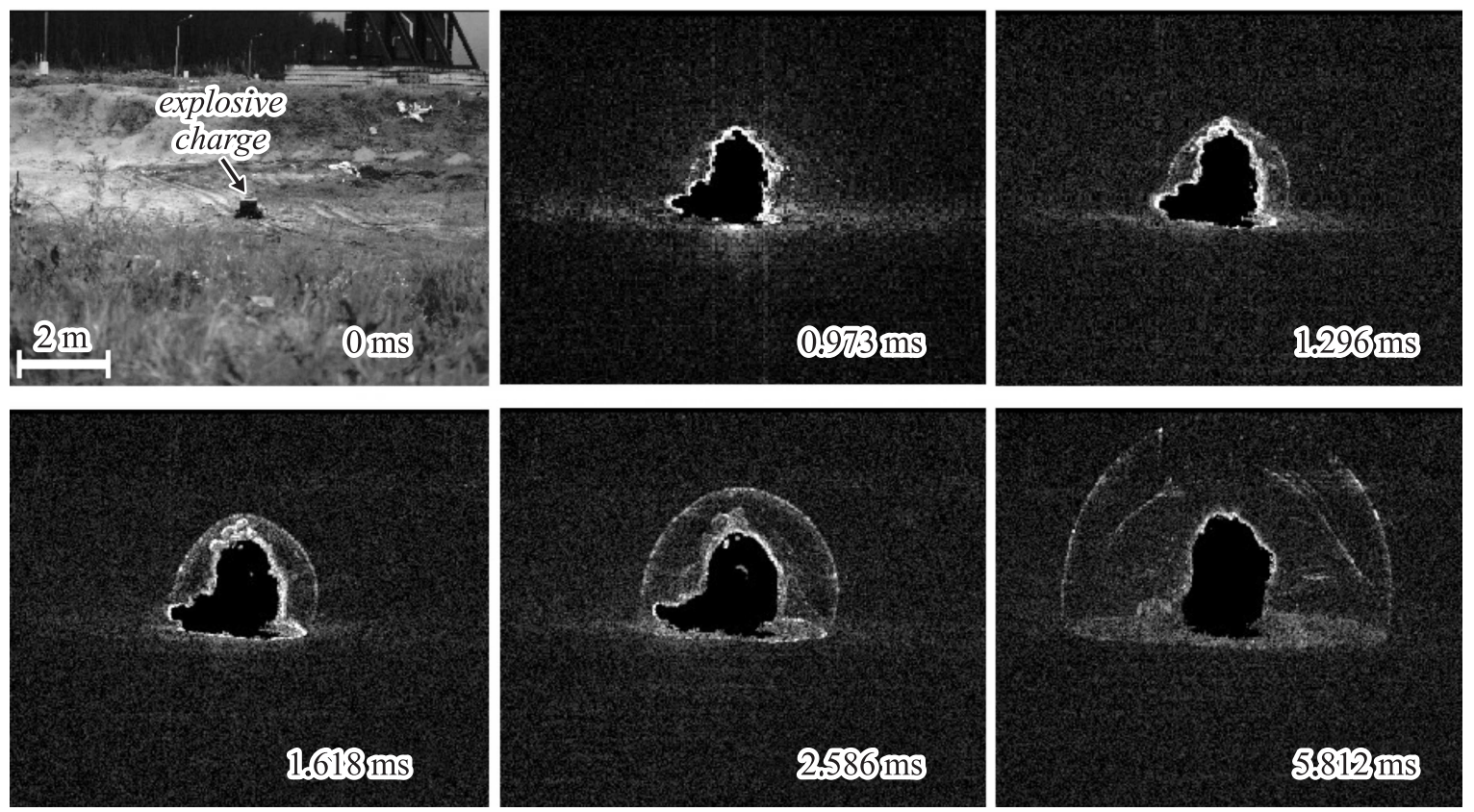

Рис. 7. Взрыв заряда ВВ цилиндрической формы с удлинением 4.3. через 0, 0.973, 1.296, $1.618,2.586,5.812 \mathrm{~ms}$ после инициирования.

рис. 8 приведен пример визуализации УВ, образованной движением ОИ по рельсовым направляющим ракетного трека со скоростью $352 \mathrm{~m} / \mathrm{s}$ [8].

\section{Измерение параметров ударных волн}

Одним из методов определения параметров УВ является анализ скорости распространения фронта первичной УВ с использованием зависимости (5), основанной на трудах Ренкина и Гюгонио [9]

$$
\Delta P=\frac{2 \rho_{0}}{k+1}\left(D^{2}-c_{0}^{2}\right),
$$

где $\Delta P$ - избыточное давление на фронте УВ $(\mathrm{Pa}), \rho_{0}-$ плотность воздуха в невозмущенной области атмосферы $\left(\mathrm{kg} / \mathrm{M}^{3}\right), k=1.4$ - коэффициент адиабаты Пуассона для воздуха, $D$ - скорость фронта УВ $(\mathrm{m} / \mathrm{s}), c_{0}$ - скорость звука в невозмущенной области $(\mathrm{m} / \mathrm{s})$.

Есть несколько способов, с помощью которых могут быть определены времена прихода УВ на ряде известных 

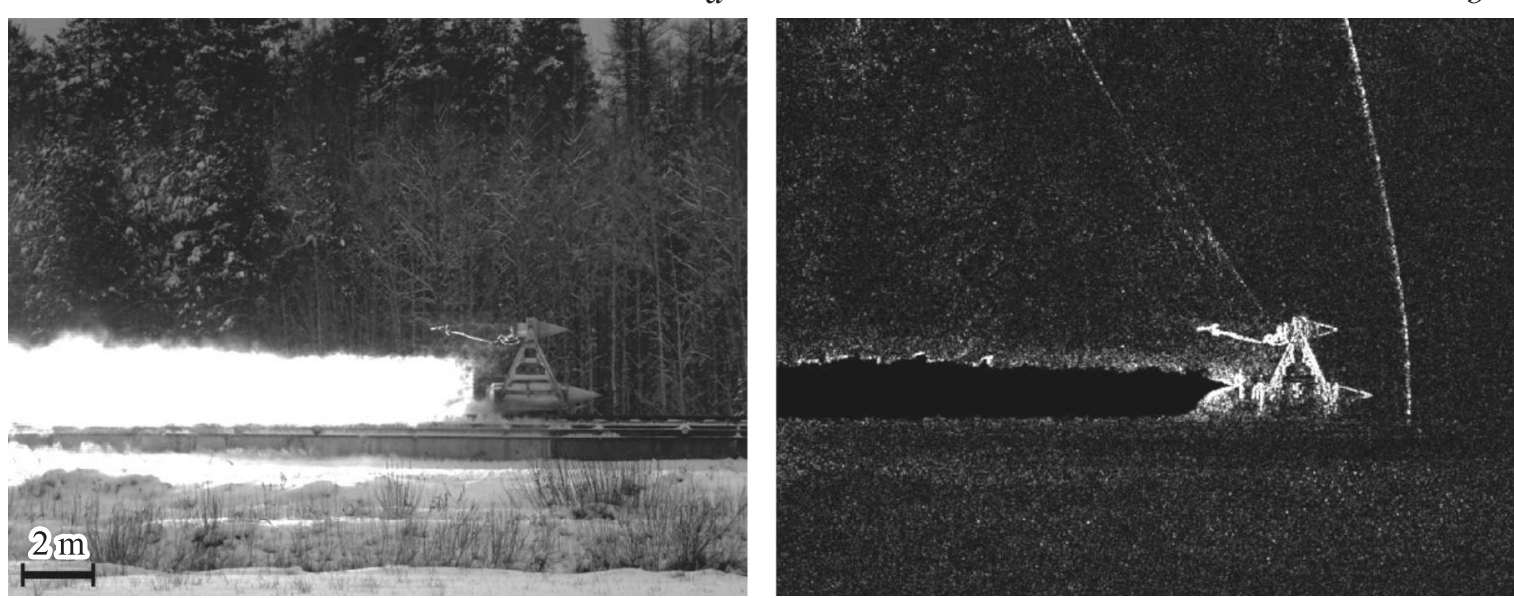

Рис. 8. Исходное изображение $(a)$ и результат визуализации УВ $(b)$.

расстояний от центра заряда. Самый большой объем информации о перемещении УВ можно получить путем высокоскоростной видеорегистрации (массив данных $R(t))$. Для того, чтобы использовать эти данные для расчета параметров УВ, необходимо вычислить скорость УВ. При непрерывном наборе дискретных данных $R(t)$ скорость УВ в любом положении зоны регистрации может быть найдена путем дифференцирования аппроксимирующей зависимости исходных данных о перемещении УВ, найденной по методу наименьших квадратов. Скорость УВ с момента, как она отрывается от продуктов детонации (число Маха $\approx 3$ ), монотонно уменьшается с увеличением радиуса и в пределе становится равной скорости звука [10]. Это обстоятельство накладывает ограничения на уравнение аппроксимирующей зависимости, так как полиномиальное уравнение не подходит для решения данных задач. В работе [11] предложено аппроксимирующее уравнение (6) для этих целей

$$
R(t)=A+B c_{0} t=C \ln \left(1+c_{0} t\right)+D \sqrt{\ln \left(1+c_{0} t\right)},
$$

где $A, B, C$ и $D-$ коэффициенты аппроксимации; $c_{0}-$ скорость звука в невозмущенной среде.

Для сильных УВ с числом Маха больше 3.5, что соответствует избыточному давлению на фронте УВ больше чем $13 \mathrm{~atm}$. Зависимость (5) не выполняется и необходимо использовать уравнения реального газа [10].

С целью отработки ТФМ для регистрации параметров УВ были проведены эксперименты, в которых проводились как высокоскоростная видеорегистрация, так и регистрация с помощью датчиков давления (ДД).

На рис. 9-12 приведены результаты одного из таких опытов. На рис. 9 представлена схема расположения регистрирующего оборудования. В опыте производился подрыв заряда 3 из прессованного тротила массой $260 \mathrm{~g}$, расположенного на деревянной подставке. Регистрация УВ, созданной при инициировании заряда, осуществлялась с помощью ДД 2 и высокоскоростной

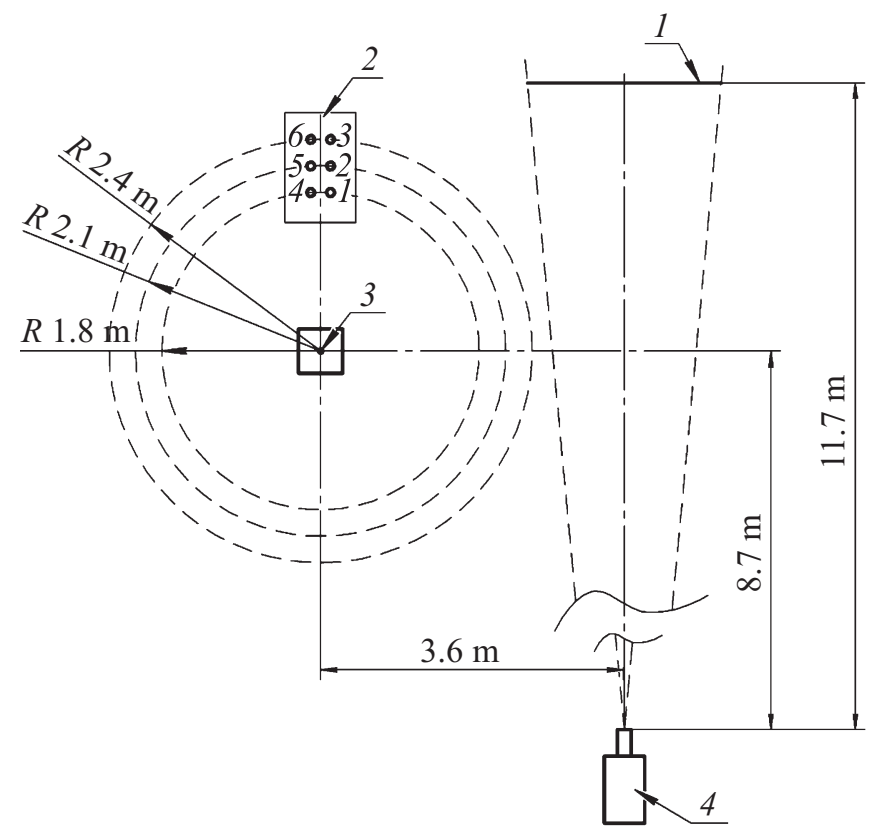

Рис. 9. Схема регистрации УВ.

видеокамеры 1. Видеорегистрация осуществлялась на фоне контрастного экрана 1 шириной $2 \mathrm{~m}$ и высотой 1 . ДД размещались в горизонтальной плоскости заряда с целью измерения избыточного давления во фронте проходящей УВ. Измерения параметров УВ, используя ДД (XCQ-080-25PSI), проводились по аттестованной методике измерений с заявленной погрешностью измерений $\pm 5 \%$.

На рис. 10 представлены один из кадров видеорегистрации и результат визуализации УВ. Визуализированы как первичная 1, так и вторичная УВ 2'. Используя результаты визуализации дискретных положений УВ и зная расположение элементов схемы оптической регистрации, найдена диаграмма перемещения фронта пер- 


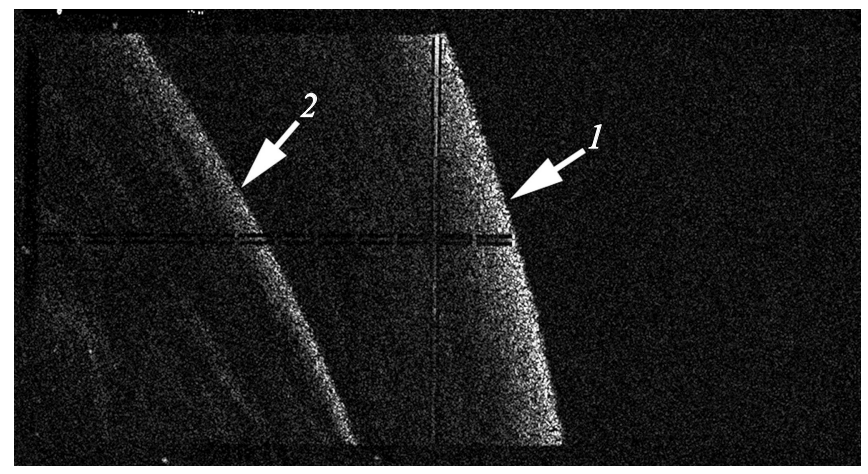

Рис. 10. Визуализация УВ в момент времени $7.6 \mathrm{~ms}$ после подрыва.

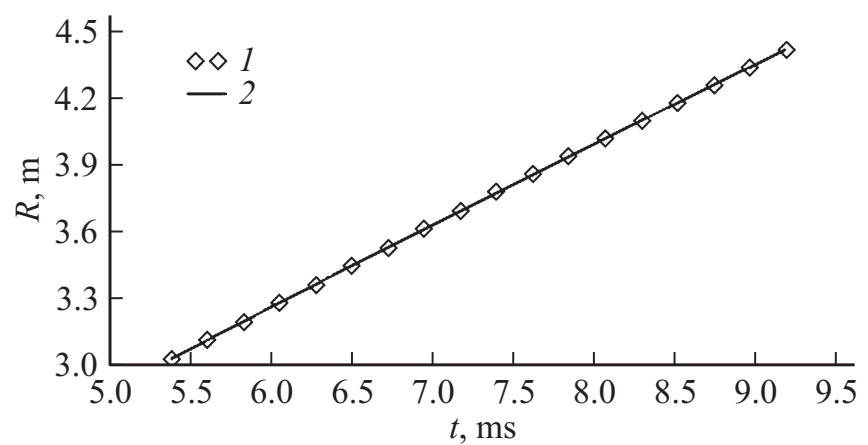

Рис. 11. $R(t)$ диаграмма распространения первичной УВ.

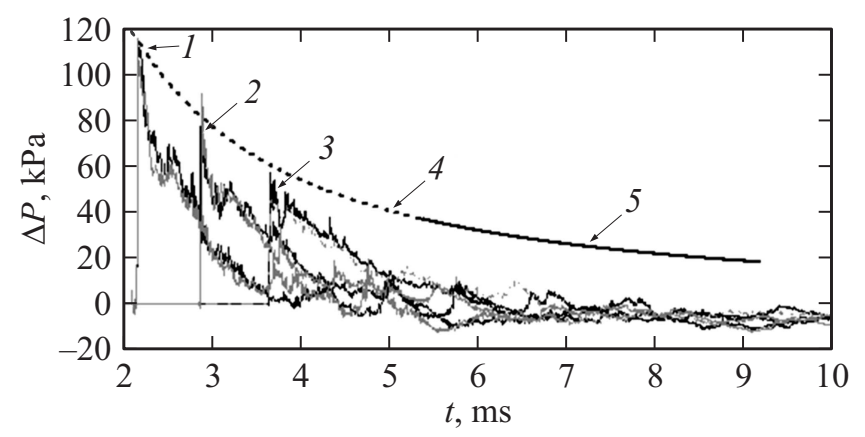

Рис. 12. Профили давлений (1)-(3) и зависимости, полученные путем пересчета $R(t)$ диаграммы (4), (5).

вичной УВ $R(t) . R(t)$ диаграмма перемещения фронта УВ приведена на рис. 11, где 1 - экспериментальные значения радиуса фронта УВ в дискретные моменты времени, 2 - аппроксимирующая зависимость. $\mathrm{B}$ качестве аппроксимирующей зависимости использовалась функция вида (6). Коэффициенты аппроксимации для приведенного случая соответствовали: $A=0.005$, $B=1.008, C=-0.426, D=1.661$. Используя аппроксимирующую зависимость $R(t)$, путем дифференцирования найдена скорость распространения УВ, и по формуле (5) произведена оценка избыточного давления во фронте первичной УВ.
Зависимости, полученные с помощью ДД, представлены на рис. 12, где 1 - информация с ДД1, ДД4; 2 - информация с ДД2, ДД5; 3 - информация с ДД3, ДД6. На данном графике также представлены зависимости, полученные по результатам оптической регистрации (4 и 5). Согласно схеме регистрации (рис. 9), ДД и видеокамера регистрировали УВ в разных, не пересекающихся, областях пространства. Кривая 4 является прогностической, она найдена с использованием аппроксимирующей зависимости $R(t)$, однако полученные результаты показывают хорошее согласование с результатами ДД.

\section{Заключение}

Приведенные данные показывают возможность использования ТФМ для регистрации УВ. Показана возможность использования ТФМ в практике проведения полигонных испытаний. ТФМ позволяет получать не только качественную, но и количественную информацию. Продемонстрирована возможность измерения параметров УВ с помощью ТФМ, достоверность получаемых результатов подтверждена с помощью ДД. В результате применения данного метода визуализируется полная картина исследуемой области (первичные, вторичные и отраженные ударные волны), что существенно повышает информативную отдачу при проведении различных экспериментов.

Работа выполнена при поддержке Программы повышения конкурентоспособности НИЯУ МИФИ.

\section{Список литературы}

[1] Meier G. // Exp. Fluid. 2002. Vol. 33. N 1. P. 181-187.

[2] Raffel M. // 2015. Exp. Fluid. Vol. 56. N 3. P. 1-17.

[3] Герасимов С.И., Файков Ю.И. // Теневое фотографирование в расходящемся пучке света. Монография. Саров: ФГУП „РФЯЦ-ВНИИЭФ“, 2010. 344 с.

[4] Бойко В.М., Оришич А.М., Павлов А.А., Пикалов А.А. Методы оптической диагностики а аэрофиическом эксперименте. Новосибирск: НГУ, 2009. 450 с.

[5] Миронова T.В. Автореф. канд. дис. Анализ деформаций, оптических неоднородностей и дисторсионных искажений с помощью искусственных спеклов в цифровой фотографии. Москва, 2012.

[6] Hargather M.J., Settles G.C. // Exp. Fluid. 2010. Vol. 48. N 1. P. 59-68.

[7] Герасимов С.И., Илюшин М.А., Кузьмин В.А. // Письма в ЖТФ. 2015. Т. 41. Вып. 7. С. 66-72.

[8] 8 Герасимов С.И., Бутова С.В. // Научная визуализация. 2015. T. 7. № 2. С. 12-20.

[9] Физика взрыва / Под ред. Л.П. Орленко. Изд. 3-е, испр., в 2 т. Т. 1. М.: Физматилит, 2004. 832 с.

[10] Dewey J.M. Measurement of the Physical Properties of Blast Waves. Ozer Igra. Experimental Methods of Shock Wave Research. 2016. P. 53-86.

[11] Dewey J.M. // Proc. Roy. Soc. Lond. A. 1971. Vol. 324. P. 275-299. 